\title{
A Continuously Infused Microfluidic Radioassay System for the Characterization of Cellular Pharmacokinetics
}

\author{
Zhen Liu*1, Ziying Jian*1, Qian Wang ${ }^{1}$, Tao Cheng ${ }^{1}$, Benedikt Feuerecker ${ }^{1}$, Markus Schwaiger ${ }^{1}$, Sung-Cheng Huang ${ }^{2}$, \\ Sibylle I. Ziegler ${ }^{1}$, and Kuangyu $\mathrm{Shi}^{1}$ \\ ${ }^{I}$ Department of Nuclear Medicine, Technische Universität München, Munich, Germany; and ${ }^{2}$ Department of Molecular and Medical \\ Pharmacology, David Geffen School of Medicine, University of California, Los Angeles, California
}

\begin{abstract}
Measurement of cellular tracer uptake is widely applied to learn the physiologic status of cells and their interactions with imaging agents and pharmaceuticals. In-culture measurements have the advantage of less stress to cells. However, the tracer solution still needs to be loaded, unloaded, and purged from the cell culture during the measurements. Here, we propose a continuously infused microfluidic radioassay (CIMR) system for continuous in-culture measurement of cellular uptake. The system was tested to investigate the influence of the glucose concentration in cell culture media on ${ }^{18} \mathrm{~F}-$ FDG uptake kinetics. Methods: The CIMR system consists of a microfluidic chip integrated with a flow-control unit and a positron camera. Medium diluted with radioactive tracer flows through a cell chamber continuously at low speed. Positrons emitted from the cells and from tracer in the medium are measured with the positron camera. The human cell lines SkBr3 and Capan-1 were incubated with media of 3 different glucose concentrations and then measured with ${ }^{18} \mathrm{~F}-\mathrm{FDG}$ on the CIMR system. In addition, a conventional uptake experiment was performed. The relative uptake ratios between different medium conditions were compared. A cellular 2-compartment model was applied to estimate the cellular pharmacokinetics on CIMR data. The estimated pharmacokinetic parameters were compared with expressions of glucose transporter-1 (GLUT1) and hexokinase-2 measured by quantitative real-time polymerase chain reaction. Results: The relative uptake ratios obtained from CIMR measurements correlated significantly with those from the conventional uptake experiments. The relative SDs of the relative uptake ratios obtained from the CIMR uptake experiments were significantly lower than those from the conventional uptake experiments. The fit of the cellular 2-compartment model to the ${ }^{18} \mathrm{~F}-\mathrm{FDG}$ CIMR measurements was of high quality. For $\mathrm{SkBr} 3$, the estimated pharmacokinetic parameters $k_{1}$ and $k_{3}$ were consistent with the messenger RNA expression of GLUT1 and hexokinase-2: culturing with low glucose concentrations led to higher GLUT1 and hexokinase-2 expression as well as higher estimated $k_{1}$ and $k_{3}$. For Capan-1, the estimated $k_{1}$ and $k_{3}$ increased as the glucose concentration in the culture medium decreased, and this finding did not match the corresponding messenger RNA expression. Conclusion: The CIMR system captures dynamic uptake within the cell culture and enables estimation of the cellular pharmacokinetics.
\end{abstract}

\footnotetext{
Received Nov. 9, 2015; revision accepted Feb. 8, 2016.

For correspondence or reprints contact: Zhen Liu, Department of Nuclear Medicine, Klinikum rechts der Isar, Technische Universität München, Ismaningerstrasse 22, 81675 Munich, Germany.

E-mail: liu@Irz.tum.de

${ }^{*}$ Contributed equally to this work.

Published online Jun. 30, 2016.

COPYRIGHT (c) 2016 by the Society of Nuclear Medicine and Molecular Imaging, Inc.
}

Key Words: microfluidic radioassay; positron imaging; cellular pharmacokinetics; glycolysis

J Nucl Med 2016; 57:1548-1555

DOI: 10.2967/jnumed.115.169151

$\mathbf{T}$ he investigation of molecular uptake inside cells is valuable for the understanding of physiologic status $(1,2)$, for the interpretation of molecular imaging results $(3,4)$, and for the development of imaging agents and pharmaceuticals (5). The cellular uptake of a molecule is usually assessed by measuring labeling signals such as radioactivity $(2,6)$, optical signal (7), or magnetic signal (8). Radiometric measurement of cellular uptake is a typical experiment in nuclear medicine (6) and provides high sensitivity for a very small mass of tracer. The assay offers the opportunity to study tracer behavior before translation to clinical applications. However, this method requires separation of the cells from the incubation medium to measure radioactivity in the cells only. During this process, the cells are processed and removed from the culture environment. Thus, only a single radioassay for a given cell-culture sample can be performed (6). A high-resolution radioluminescence microscope was developed by Pratx et al. $(2,9)$. It allows uptake by a single cell to be measured by culturing cells in a thin scintillator and imaging them using invert microscopy with an electron-multiplying chargecoupled-device camera (9). In another approach, the automatic measurement of real-time tracer binding was achieved on a radioimmunoassay system using a rotating cell dish (10). Vu et al. developed a microfluidic radioassay system (BetaBox) that integrates a microfluidic chip with a position-sensitive $\beta$-camera (11). That system allows multiple radioassays of a small cell population directly in a culture environment. The cell cultures can be maintained for long periods, and changes in the environment can be digitally controlled, which is convenient for the investigation of diagnostic and therapeutic radiopharmaceuticals. The BetaBox radioassay system has been used as a platform to rapidly screen the response of various cell lines to mechanistically distinct, targeted drugs $(12,13)$. A cellular 2-compartment model was developed to quantify cellular pharmacokinetics based on discrete measurements on the BetaBox system (14).

Although the advantages of this pioneering microfluidic radioassay system are promising, it requires loading and unloading of the incubating tracers for each assay. Thus, the measurements are restricted to several discontinuous time points, and a dynamic acquisition over time is still not possible. In addition, the medium-exchange 
procedure requires a compromise between the completeness of the exchange and maintenance of a stable culture environment $(11,12)$. Application of this technique to less adhesive cell lines is therefore limited. Also, the amount of residual medium left in the cell chamber is difficult to check.

In this study, we developed a continuously infused microfluidic radioassay (CIMR) system to acquire dynamic information on cellular uptake and improve the quantification of cellular tracer kinetics.

${ }^{18}$ F-FDG PET is widely used for in vivo tumor imaging based on the increased glycolysis in tumor cells known as the Warburg effect (15-17). Uptake of ${ }^{18} \mathrm{~F}$-FDG is considered to be biomarker of tumor malignancy and of prognostic value for therapy management. After entering cells via glucose transporters (GLUTs), ${ }^{18} \mathrm{~F}-\mathrm{FDG}$ is phosphorylated by hexokinase and becomes trapped in the cells. When ${ }^{18} \mathrm{~F}-\mathrm{FDG}$ is used for tumor diagnosis and for investigation of therapy response, uptake of ${ }^{18} \mathrm{~F}-\mathrm{FDG}$ is usually interpreted with regard to the expression of GLUTs and hexokinases in the glycolytic pathway. Among these two families of proteins, GLUT1 and hexokinase-2 have been investigated the most. GLUT1 expression is usually considered to be associated with malignant tumor stages (18). ${ }^{18} \mathrm{~F}-\mathrm{FDG}$ uptake has been shown to be more influenced by GLUT1 than by the other subtypes of GLUT in many tumor tissues, such as breast cancer (19), pancreatic tumor (20), and cervical cancer (21). Hexokinase-2 directly mediates glycolysis and promotes tumor growth (22) and has been found to be the predominant isoform of hexokinases in many tumors $(23,24)$. Overexpression of hexokinase- 2 has been reported to increase ${ }^{18} \mathrm{~F}-\mathrm{FDG}$ uptake in cancer cells (25). GLUT1 and hexokinase-2 have been used as a therapeutic target for drug development associated with cancer metabolism (26). On the other side, the expression of GLUT1 and hexokinase-2 varies among different types of cancer cells (27), and tumors possess the special quality of being able to adjust the expression of GLUT1 or hexokinase-2 to maintain their energy supply and homeostasis (25). However, it is difficult to investigate the adaption of their functions under various metabolic conditions $(28,29)$.

As a proof of the concept of the proposed CIMR system, we focused on the investigation of ${ }^{18} \mathrm{~F}-\mathrm{FDG}$ uptake in tumor cells. The applicability of the proposed CIMR system was verified by comparing the estimated ${ }^{18} \mathrm{~F}$-FDG kinetics with literature data and the corresponding expression of GLUT1 and hexokinase-2 measured with conventional quantitative polymerase chain reaction (qPCR).

\section{MATERIALS AND METHODS}

\section{CIMR System}

The CIMR system is based on a microfluidic chip ( $\mu$-Slide $\mathrm{VI}^{0.4}$; ibidi $\mathrm{GmbH}$ ), a flow control unit, and a positron camera as shown in Figure 1. The microfluidic chip consists of two parallel chambers $(17 \times 3.8 \times$ $0.4 \mathrm{~mm}$ each), each with a separate inlet and outlet. The two chambers are connected as shown in Figure 1B. One serves as a medium-monitoring chamber and the other as a cell culture chamber. The flow control unit is a programmable syringe pump (Cavro XLP 6000; Tecan Group Ltd.) with connecting flow tubes. The medium with radioactive tracer is driven by the pump from the reservoir via the monitoring chamber into the culture chamber (Fig. 1B). The monitoring and culture chambers are simultaneously measured by a positron camera (Fig. 1C), which consists of a single-particle-counting silicon pixel detector (300 $\mu \mathrm{m}$ thick, Crypix; Crytur Ltd.) bonded to a complementary metal oxide-semiconductor readout chip (TimePix; European Organization for Nuclear Research). The field of view of the positron camera is $14 \times 14 \mathrm{~mm}(256 \times 256$ pixels). Details on the basic performance of this detector for direct positron measurement have been previously published (30).

\section{Cell Experiments}

Cell Culture Preparation. Two types of human cancer cell lines, Capan-1 (pancreas adenocarcinoma) and $\mathrm{SkBr} 3$ (breast adenocarcinoma), were selected for the investigation. Two days before the CIMR measurements, approximately $1.5 \times 10^{4}$ cells in a $30-\mu \mathrm{L}$ single-cell suspension were inoculated into the cell culture chamber of a microfluidic chip using a pipette, to form a homogeneous single layer on the bottom surface of the chamber. The cells were then cultured in Dulbecco modified Eagle medium with a $25 \mathrm{mM}$ glucose concentration (Biochrom $\mathrm{GmbH}$ ), supplemented with fetal bovine serum (10\%), penicillin $(100 \mathrm{U} / \mathrm{mL})$, and streptomycin $(100 \mu \mathrm{g} / \mathrm{mL})$ under controlled conditions of $37^{\circ} \mathrm{C}$ and $5 \% \mathrm{CO}_{2}$. One day before the measurements, the medium in the cell chamber was switched to 1 of 3 special media with a $5,2.5$, or $0.5 \mathrm{mM}$ glucose concentration. The corresponding medium was exchanged every $8 \mathrm{~h}$ until the start of the measurements to sustain a stable glucose concentration. At the time of the CIMR measurements, the cells had reached approximately $60 \%-90 \%$ confluence inside the cell chamber.

Measurements with the CIMR System. Before the infusion, the flow control unit was sterilized and cleaned using $70 \%$ ethanol and ultrapure water. ${ }^{18} \mathrm{~F}-\mathrm{FDG}$ was diluted into the cell culture medium to generate a radioactive solution of $0.2-5 \mathrm{MBq} / \mathrm{mL}$ with the investigated glucose concentration. The tracer medium solution was pumped through the medium chamber and cell chamber at a constant flow of $1.25 \mu \mathrm{L} / \mathrm{s}$, which created a small shear stress $\left(<0.13 \mathrm{dyn} / \mathrm{cm}^{2}\right.$, estimated on the basis of the application note of ibidi $\mathrm{GmbH}$ ) near the chamber surface. This flow speed leads to a medium refresh rate of 2.5 times per minute, which is sufficient to maintain the continuousinfusion profile. Positrons emitted both from the medium chamber and from the cell chamber were measured by the positron camera for $65 \mathrm{~min}$. The measurements were binned into frames of $1 \mathrm{~min}$ each. Each measurement was repeated 6 times.

After the CIMR measurements, the cell chamber was imaged using a microscope (BZ9000; Keyence Co. Ltd.) with a phase-contrast objective lens of $\times 20$. Five widely distributed microscopic fields of view were selected for counting the number of cells. The average
FIGURE 1. Setup of CIMR system: photograph of microfluidic chip in operation (A), diagram of tracer medium flow during measurement $(B)$, side view of cell chamber during measurement, at the cut point shown in $B(C)$, and example frame from positron camera during CIMR measurement (D).

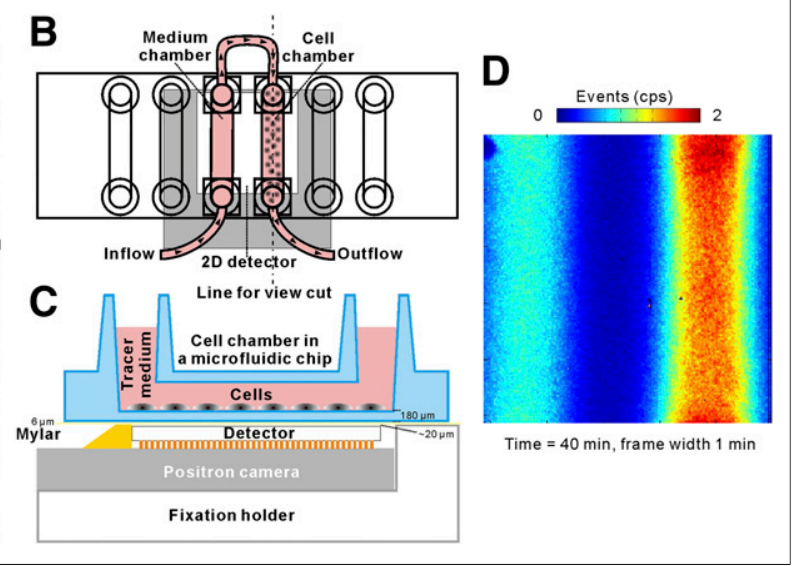


number of cells in the 5 fields of view was then normalized to the cell number per detector pixel $(55 \times 55 \mu \mathrm{m})$.

Conventional Uptake Measurement. As previously described (6), ${ }^{18} \mathrm{~F}-$ FDG uptake by cells was studied with the conventional uptake method for each of the culture conditions used in the CIMR measurements. Two days before the uptake experiment, approximately $6 \times 10^{4}$ cells in a $300-\mu \mathrm{L}$ suspension were inoculated into one well of a 24-well plate. One day before the uptake experiment, the cells were incubated in medium containing 1 of the 3 glucose concentrations used in the CIMR studies. Medium was refreshed every $8 \mathrm{~h}$ before the uptake measurement. During the uptake measurement, an ${ }^{18} \mathrm{~F}-\mathrm{FDG}$ solution with the same glucose concentration as used in the corresponding CIMR measurements was applied to the cells for 4 different periods (10,20,30, and $40 \mathrm{~min})$. Then, the cells were washed twice with ice-cold phosphate-buffered saline buffer and dissociated by trypsin/ethylenediamine tetraacetic acid solution (Biochrom $\mathrm{GmbH})$. The radioactivity of the collected cells in each well was measured using a $\gamma$-counter (Wallac Wizard 1470; PerkinElmer). Thus, 3 repeated measurements of ${ }^{18} \mathrm{~F}-\mathrm{FDG}$ uptake for incubation times of 10, 20, 30, and $40 \mathrm{~min}$ were obtained for each medium condition. The number of collected cells in each well was counted using an automated cell counter (Countess; Invitrogen Life Technologies $\mathrm{GmbH}$ ). The counted cell numbers of the wells with the same culture condition on the same day were averaged.

$q P C R$. Cells under the same conditions as in the CIMR measurements were cultured for molecular biology assays. Total RNA was extracted from each tumor cell line using the RNeasy Plus Mini kit (Qiagen); complementary DNA was prepared from the total RNA isolated using the QuantiTect reverse-transcription kit (Qiagen). The sequences of primers for reverse-transcription PCR were as follows. For GLUT1 (31), forward was 5'-CAG GAG ATG AAG GAA GAG-3' and reverse was $5^{\prime}$-TCG TGG AGT AAT AGA AGA C-3'. For hexokinase2 (22), forward was 5'-CAA AGT GAC AGT GGG TGT GG-3' and reverse was CAA AGT GAC AGT GGG TGT GG-3'. For the housekeeping gene, forward was 5' -CAG ATG GCA AGA CAG TAG AAG-3 and reverse was 5'-GGC AAA AAT GGA AGC AAT GG-3'. All primers were synthesized by Eurofins Genomics. qPCR was performed on LightCycler 480 Instrument I/II (Roche Diagnostics) with the LightCycler 480 SYBR Green I Master kit, and the data were analyzed by LightCycler 480 software, version 1.05.0.39.

\section{Data Processing and Analysis}

Image Processing and Normalization. The acquired dynamic data were corrected for radioactive decay. For each 1-min frame, the mean events per pixel $(55 \times 55 \mu \mathrm{m})$ within a box region of interest $(12 \times 3.8 \mathrm{~mm})$ in the center of an imaged chamber were calculated separately for the medium chamber and for the cell chamber. Time-activity curves were generated to describe changes in mean events per pixel versus time. The calibrated radioactivity of the medium in the cell chamber was estimated on the basis of the measured time-activity curve of the medium chamber after correction of delay and dispersion. This can be described using the following formula (32):

$$
\beta_{\mathrm{cm}}(t)=\beta_{\mathrm{m}}(t-\Delta T) * \frac{1}{\tau} e^{-\frac{t}{\tau}}
$$

where $\beta_{\mathrm{cm}}$ and $\beta_{\mathrm{m}}$ are, respectively, the event density curves of the medium in the cell chamber and the medium chamber; $\Delta T$ and $\tau$ are, respectively, coefficients characterizing the delay and dispersion of the ${ }^{18} \mathrm{~F}-\mathrm{FDG}$ activity flowing from the medium chamber to the cell chamber; and * is the convolution operator.

To estimate the delay and dispersion coefficients, the CIMR system was run 6 times without cells in the cell chamber using the same fluidic condition as in the real measurements. The coefficients of the decay and dispersion parameters were determined regularly, especially after the settings were changed for the CIMR operating condition.
The microfluidic chamber has a height of $400 \mu \mathrm{m}$, and the positron camera is placed below the chamber. Thus, the positron camera had lower sensitivity for the positrons emitted from upper layers than from lower layers in the chamber. This depth-dependent sensitivity profile of the positron camera was corrected in the measurements by multiplying the event density by a depth-dependent sensitivity correction factor of 1.74 (derived as described in the supplemental file and shown in Supplemental Fig. 1; available at http://jnm.snmjournals.org).

The events for the cells in the cell chamber were calculated by subtracting the estimated events in the medium in the cell chamber from the total measured events in the cell chamber. The ${ }^{18} \mathrm{~F}-\mathrm{FDG}$ concentration in the medium was computed by normalization to the volume associated with a pixel in the chamber $(55 \times 55 \times 400 \mu \mathrm{m})$. The cellular ${ }^{18} \mathrm{~F}$-FDG concentration was calculated using an estimated mean cell number and mean cell volume (Supplemental Fig. 2). The mean volume of adherent cells with mean diameter $d$ was approximated by the volume of an ellipsoid $\left(\pi d^{3} / 12\right)$. Cellular uptake was estimated as the ratio of cellular ${ }^{18} \mathrm{~F}$ FDG events (cps) per $10^{6}$ cells over the corresponding density of ${ }^{18} \mathrm{~F}-\mathrm{FDG}$ events in the medium (cps/mL) (Supplemental Fig. 2). The relative uptake ratios from the CIMR system were calculated as the ratios between the uptake time-activity curves of the corresponding culture conditions for each time point. Similarly, the relative uptake ratios for the conventional uptake experiment were calculated as the ratios between uptake values at the discrete measurement times. In this study, the uptake ratios relative to the ratio for the $5 \mathrm{mM}$ culture medium were calculated. All processing was done using Matlab, version 2012b (The MathWorks, Inc.).

Cellular Pharmacokinetic Modeling. On the basis of the time-activity curves obtained from the CIMR measurement, the pharmacokinetic parameters related to GLUT and hexokinase can be estimated. For ${ }^{18} \mathrm{~F}-\mathrm{FDG}$, the uptake is controlled by GLUT and hexokinase phosphorylation (Supplemental Fig. 3A). A cellular 2-compartment model was constructed to estimate the kinetic parameters relating to ${ }^{18} \mathrm{~F}-\mathrm{FDG}$ transport and phosphorylation (Supplemental Fig. 3B) (14). Given the event density in the medium $\beta_{\mathrm{cm}}$, the free ${ }^{18} \mathrm{~F}-\mathrm{FDG}$ events in the cell $\beta_{\text {in }}$ and the phosphorylated ${ }^{18} \mathrm{~F}-\mathrm{FDG}$ events $\beta_{\mathrm{ph}}$ in the cell can be described as follows:

$$
\begin{aligned}
& \frac{d \beta_{\mathrm{in}}(t)}{d t}=k_{1} \beta_{\mathrm{cm}}(t)-k_{2} \beta_{\text {in }}(t)-k_{3} \beta_{\text {in }}(t)+k_{4} \beta_{\mathrm{ph}}(t) \\
& \frac{d \beta_{\mathrm{ph}}(t)}{d t}=k_{3} \beta_{\mathrm{in}}(t)-k_{4} \beta_{\mathrm{ph}}(t),
\end{aligned}
$$

where the rate constants $k_{1}, k_{2}, k_{3}$, and $k_{4}$ are, respectively, the import and export rates of ${ }^{18} \mathrm{~F}-\mathrm{FDG}$ across the cell membrane and the phosphorylation and dephosphorylation rates of ${ }^{18} \mathrm{~F}-\mathrm{FDG}$ inside the cell. The parameters were estimated by fitting the time-activity curves derived from CIMR measurements. The model fit was performed by a trust-region algorithm implemented using $\mathrm{C}++$ with the MKL library (Intel).

A Michaelis-Menten equation was applied to interpret the relation between the estimated rate constants $\left(k_{1}\right.$ and $\left.k_{3}\right)$ and the messenger RNA (mRNA) expressions of the corresponding enzyme or transporter (33):

$$
k \sim \frac{\mathrm{V}_{\max }}{K_{m}+\mathrm{C}_{\text {endo }}},
$$

where $\mathrm{C}_{\text {endo }}$ is the concentration of the endogenous substrate, $K_{m}$ is the substrate concentration at the half-maximum reaction rate, and $\mathrm{V}_{\max }$ is the maximum reaction rate, which is assumed to be proportional to protein expression. Considering that the glucose concentration of the medium was kept constant before and during CIMR, the endogenous glucose concentration was assumed to be the same as the glucose concentration of the medium. The ${ }^{18} \mathrm{~F}$-FDG concentration is negligible compared with the glucose concentration of the medium. For each cell line, we used fitting of the Michaelis-Menten equation to determine whether the $K_{m}$ rate can 

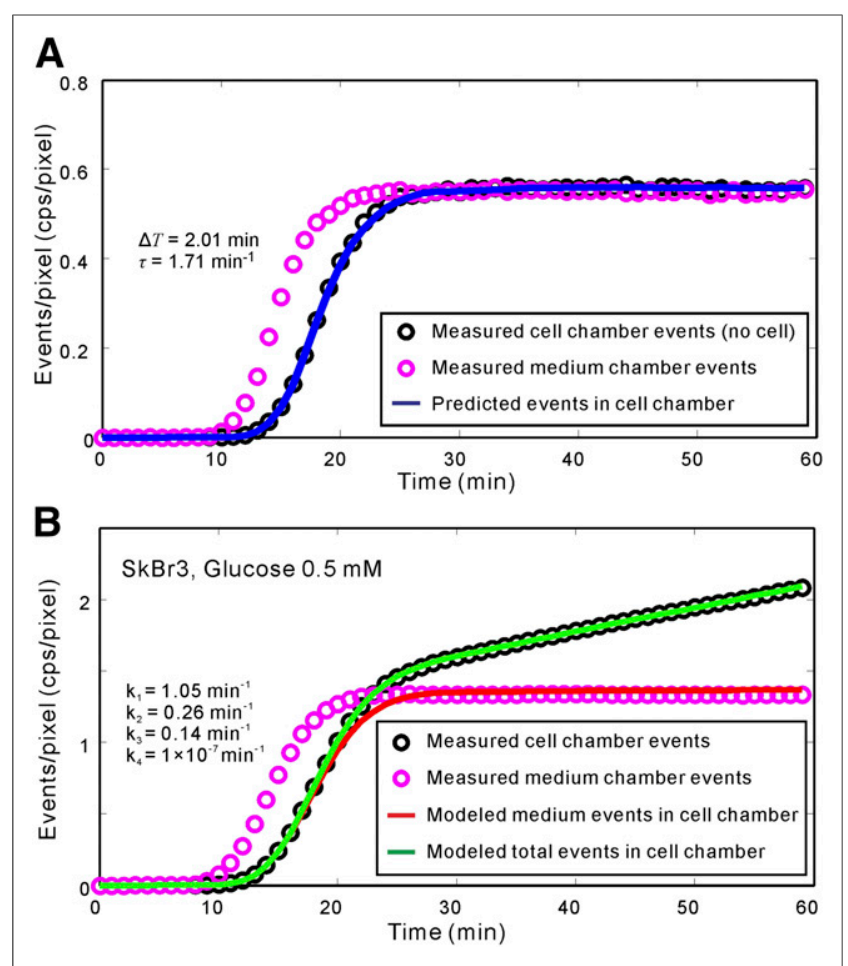

FIGURE 2. (A) Model fitting for delay and dispersion, along with curve predicted using fit results. (B) Pharmacokinetic model fitting for uptake by $\mathrm{SkBr} 3$ cells cultured in $0.5 \mathrm{mM}$ glucose.

be the same for cultures using different glucose concentrations. The nonlinear least square fit was applied to fit the cellular pharmacokinetic parameter to the mRNA expressions.

Pearson correlation was applied to compare the relative uptake ratios between the CIMR and conventional uptake experiments. The paired Student $t$ test was used to further compare the relative SD (SD/mean) of the two methods. A significance level of $P<0.05$ was established.

\section{RESULTS}

During the continuous-infusion procedure, the loss of cells was $1.3 \%-4.5 \%$ (mean $\pm \mathrm{SD}, 3.2 \% \pm 1.7 \%[n=3]$ ) for Capan- 1 and $0.9 \%-3.5 \%(1.9 \% \pm 1.4 \%[n=3])$ for $\mathrm{SkBr} 3$. The difference in sensitivity between the medium chamber and the cell chamber with no cells was $0.3 \%-1.1 \%(0.6 \% \pm 0.3 \%[n=6])$.

The modeled curves including delay and dispersion fit the measured curves well for the 6 calibration runs with no cells in the cell chamber $\left(R^{2}=0.9995 \pm 0.0003\right)$. One example is shown in Figure 2A. The estimated delay coefficient $(2.11 \pm 0.11 \mathrm{~min})$ and dispersion coefficient $\left(1.70 \pm 0.11 \mathrm{~min}^{-1}\right)$ had a small relative $\mathrm{SD}(<7 \%)$. When the mean delay and dispersion coefficients of the 6 curves were applied to predict the time-activity curve in the cell chamber, the absolute percentage error was $1.3 \% \pm 0.3 \%$ for the 6 runs (Fig. 2A).

The measured data of the CIMR were well fit by the cellular 2-compartment model; there was a high-quality fit $\left(R^{2}>0.9999\right)$ for both $\mathrm{SkBr} 3$ and Capan-1 under the investigated conditions. Figure 2B shows an example of the model fitting for $\mathrm{SkBr} 3$ cultured in medium containing $0.5 \mathrm{mM}$ glucose; the figure plots the modeled medium events in the cell chamber (after correction for delay and dispersion). After fitting using the cellular 2-compartment model, the modeled total-event curve fit well the measured total events in the cell chamber.
Figure 3 plots the mean and SD of the estimated cellular uptake (mL/10 $10^{6}$ cells) on the CIMR system for 6 repeated measurements. For the same cell under the same condition, uptake was similar. The averaged relative SDs of normalized uptake for $\mathrm{SkBr} 3$ in the 3 culture media were $18.5 \%(5 \mathrm{mM}), 15.1 \%(2.5 \mathrm{mM})$, and $5.4 \%(0.5 \mathrm{mM})$ (Fig. 3A). For Capan-1, the corresponding averaged relative SDs were $8.8 \%(5 \mathrm{mM}), 8.0 \%$ (2.5 mM), and $9.1 \%$ (0.5 mM) (Fig. 3B).

The relative uptake ratios of CIMR and conventional uptake experiments are shown in Figure 4. A significant correlation was found between the CIMR and conventional uptake experiments for $\operatorname{SkBr} 3\left(r=0.98, P=9 \times 10^{-6}\right)$ and Capan-1 $(r=0.95$, $P=0.0003)$. Overall, the CIMR experiments had a relative SD of $11.3 \%$, which is significantly less $(P=0.004)$ than the $20.8 \%$ relative $\mathrm{SD}$ found for the conventional uptake experiments.

The $k_{1}$ of the $\mathrm{SkBr} 3$ cells was about $75 \%$ higher when they were cultured with $0.5 \mathrm{mM}$ glucose than with 2.5 or $5 \mathrm{mM}$ (Fig. 5). This finding agrees with the qPCR results for GLUT1 mRNA level, in which the GLUT1 expression of cells was around $60 \%$ higher for the $0.5 \mathrm{mM}$ medium than for the 2.5 or $5 \mathrm{mM}$. Similarly, the $k_{3}$ was higher for the $0.5 \mathrm{mM}$ medium than for the 2.5 or $5 \mathrm{mM}$. The hexokinase- 2 mRNA levels of cells were higher for the $0.5 \mathrm{mM}$ medium than for the 2.5 or $5 \mathrm{mM}$.

The $k_{1}$ of the Capan- 1 cells was around $25 \%$ higher when they were cultured with $0.5 \mathrm{mM}$ glucose than with 2.5 or $5 \mathrm{mM}$ (Fig. 6). However, the qPCR results for the GLUT1 expression of cells was higher for the $5 \mathrm{mM}$ medium than for the 2.5 or $0.5 \mathrm{mM}$. The $k_{3}$ was more than $60 \%$ higher for the $0.5 \mathrm{mM}$ medium than for the 2.5 or $5 \mathrm{mM}$. However, the increase in hexokinase- $2 \mathrm{mRNA}$ levels

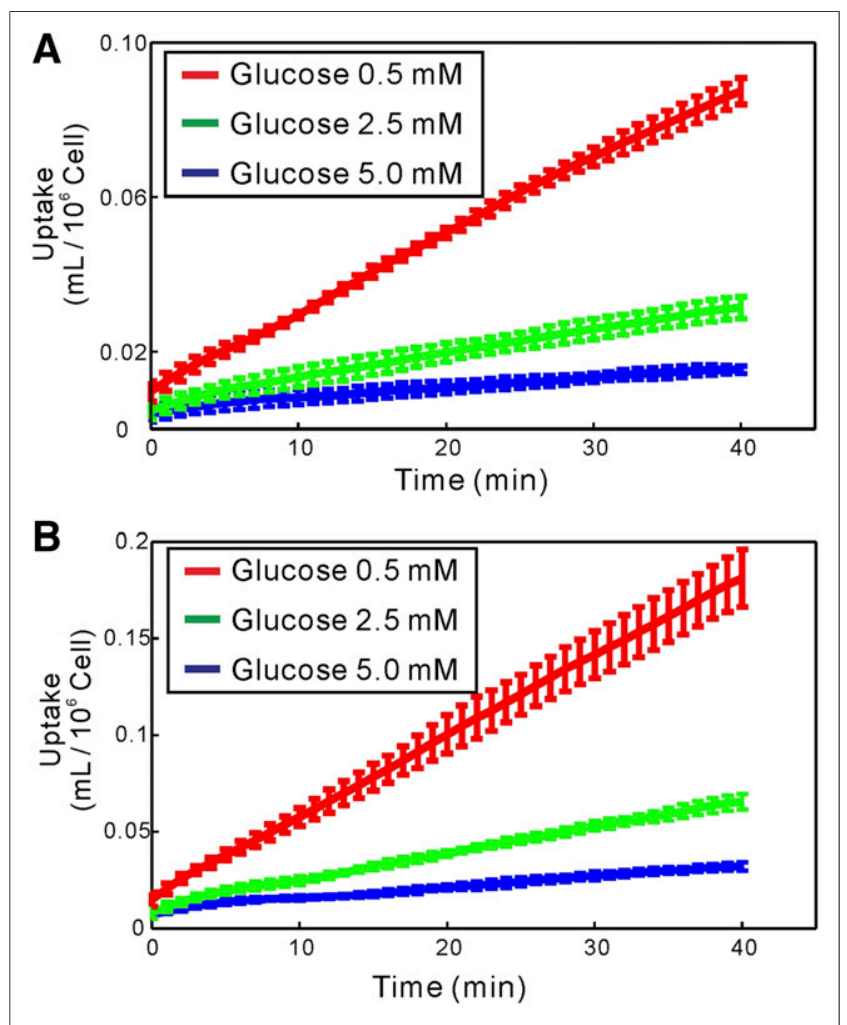

FIGURE 3. Mean and SD of normalized uptake curves plotted for 6 repeated measurements on CIMR under 3 different culturing conditions for SkBr3 (A) and Capan-1 (B). 


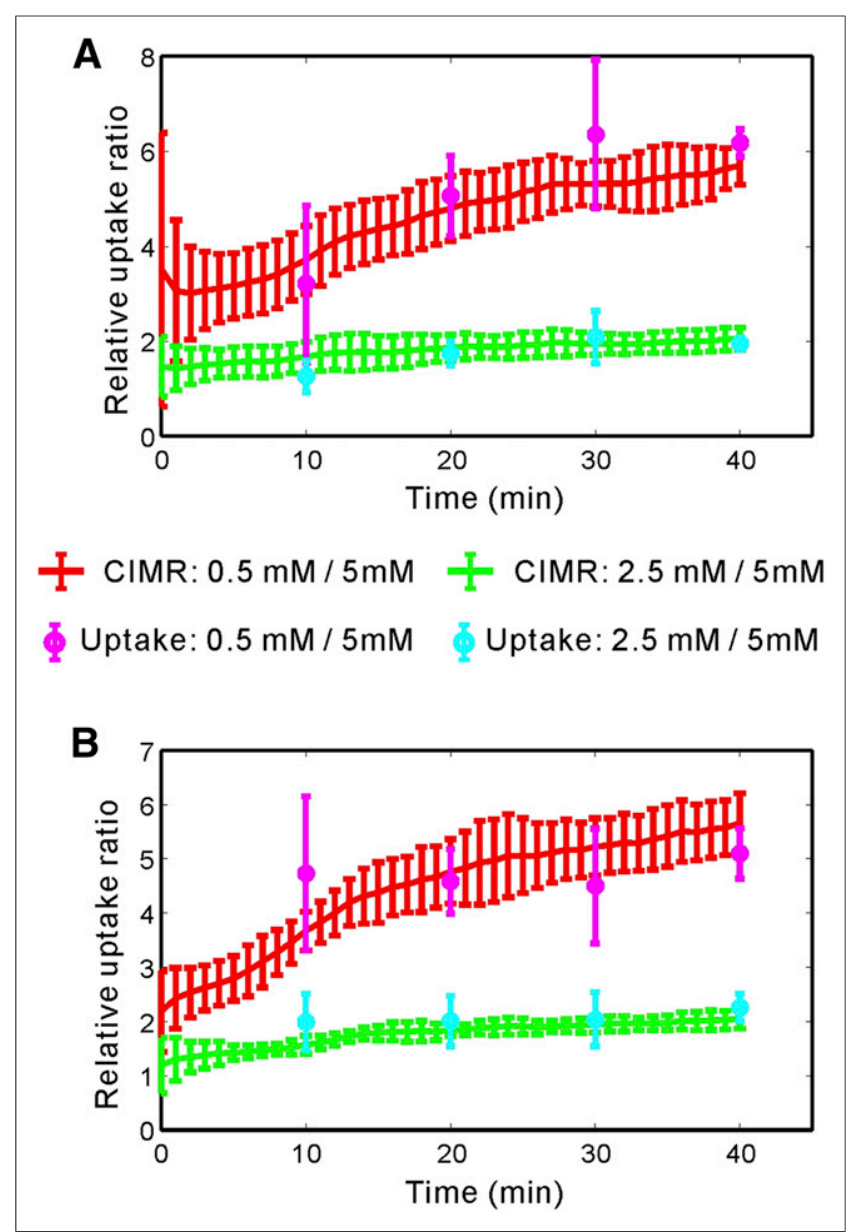

FIGURE 4. Comparison of relative uptake ratio between CIMR uptake time-activity curves and conventional measurements of uptake by SkBr3 (A) and Capan-1 (B). Mean and SD of 6 repeated measurements of each type are plotted.

was less than $25 \%$ when the $0.5 \mathrm{mM}$ medium was compared with the 2.5 and $5 \mathrm{mM}$.

For $\mathrm{SkBr} 3$, the estimated cellular kinetics could be fit to the mRNA expressions using a fixed $K_{m}$ with the determinant coefficient $\left(R^{2}=0.73\right.$ for $k_{1}$ and $R^{2}=0.97$ for $\left.k_{3}\right)$. However, for Capan- 1 , it was not possible to fit the estimated cellular kinetics to the corresponding mRNA levels using a fixed $K_{m}$.

\section{DISCUSSION}

Similar to the previous approach-BetaBox (discontinuous microfluidic radioassay) (11) — the proposed CIMR allows in-culture measurements. It does not require loading, unloading, and cleaning of the tracer medium in the culture chamber. Thus, the measurements can deliver direct cellular-uptake information without introducing additional stress during the medium exchange. In particular, the continuous measurement captures the full dynamic course of cellular uptake, enabling application of pharmacokinetic analysis. The results of these initial tests showed that this system can achieve reproducible cellular uptake measurements as well as a stable estimation of cellular kinetics. However, the volume of the microfluidic-chip chamber in our system is much larger than that in the BetaBox system. The larger volume is needed obtain a cell-uptake signal sufficient to distinguish background-medium events during the mixture measurements of cell uptake and medium.

The medium chamber in the CIMR system monitors the medium events in the cell chamber. However, the measured medium events need to be corrected for delay and dispersion before the time-activity curve can be used to correct the measurement of the cell chamber. The effective volume of the microfluidic chamber is $30 \mu \mathrm{L}$, the volume of the two connectors is $56.5 \mu \mathrm{L}$ each, and the volume of the tubing in between is $14.1 \mu \mathrm{L}$. Under the experimental flow speed, the theoretic delay between cell chamber and medium chamber is $2.09 \mathrm{~min}$. The estimated delay coefficient, $2.11 \mathrm{~min}$, agrees with the theoretic estimation.

Although the volume between the two chambers may slightly vary because the connection tubes are manually placed, the small relative SD has little influence $(<2 \%)$ on the predicted medium events of the cell chamber and can be ignored for high uptake signals.

The CIMR system operates under a continuous-infusion condition, and the fluid shear stress on cells is less than $0.13 \mathrm{dyn} / \mathrm{cm}^{2}$. Under physiologic conditions, the interstitial-flow shear stress on normal tissues is on the order of $0.1 \mathrm{dyn} / \mathrm{cm}^{2}$ or lower (34). Thus, the fluid shear stress in our system is similar to that under physiologic conditions. In addition, the low cell loss $(<4.5 \%)$ during the CIMR measurements shows that this low shear stress does not significantly influence the adherence of the two investigated cell types in the chamber. However, for less adherent cells, even a low shear stress may lead to nonnegligible cell loss. Further strategies are needed to compensate for the leaching out of these less adherent cells. Otherwise, such cells are not suitable for CIMR measurements.

The uptake values obtained in CIMR are calculated from mediumchamber and cell-chamber event densities, which are not absolute activity concentrations. The sensitivity of the system (calibration of event density to absolute activity concentration) is not considered during the calculation. We put a Mylar sheet (6 $\mu \mathrm{m}$; DuPont Teijin Films) between the microfluidic chip and the detector to prevent possible leakage of fluid onto the semiconductor detector during port

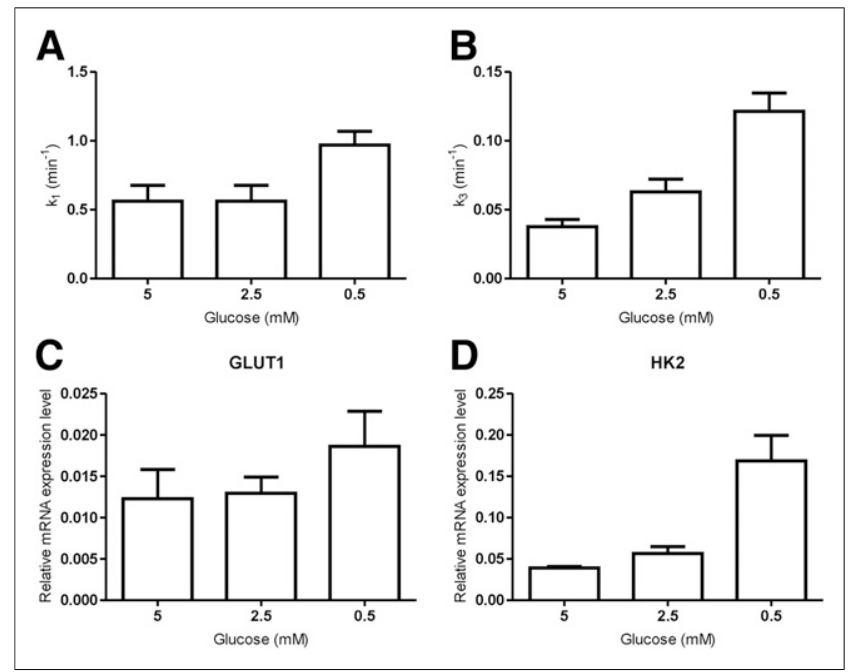

FIGURE 5. Comparison of cellular pharmacokinetics of CIMR and qPCR for $\mathrm{SkBr} 3$ in culture media with different glucose concentrations $(5,2.5$, and $0.5 \mathrm{mM}$ ). (A and B) Plots of estimated cellular pharmacokinetics $k_{1}$ and $k_{3}$ based on CIMR data. (C and D) Plots of qPCR measurements of GLUT1 and hexokinase-2 (HK2) for cells under same culture conditions. 


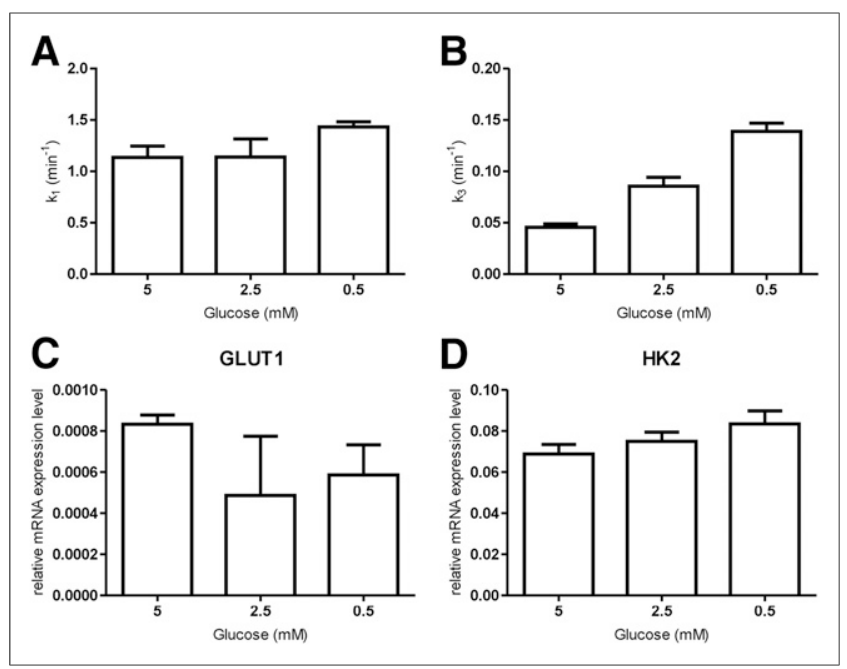

FIGURE 6. Comparison of cellular pharmacokinetics of CIMR and qPCR for Capan-1 in culture media with different glucose concentrations $(5,2.5$, and $0.5 \mathrm{mM})$. (A and B) Plots of estimated cellular pharmacokinetics $k_{1}$ and $k_{3}$ based on CIMR data. (C and D) Plots of qPCR measurements of GLUT1 and hexokinase-2 (HK2) for cells under same culture conditions.

exchange. The air space between the microfluidic chip and the detector may change in different setups, leading to a slightly varying absolute sensitivity. However, we measured the medium chamber and the cell chamber simultaneously. Thus, the sensitivity change did not affect calculation of the uptake or estimation of the kinetic parameters (as proven in the supplemental file). The difference in absolute sensitivity between the medium chamber and the cell chamber was less than $1.1 \%$ (mean, $0.6 \% \pm 0.3 \%$ ); thus, the same sensitivity was assumed for both chambers. Even after careful calibration and correction, the estimated uptake values differed from the conventional uptake values obtained from well counter measurements (Supplemental Fig. 4). This observation may be explained by the difference between in-culture measurements and conventional ex-culture measurements. The influence of the sample preparation procedure (wash with cold phosphate-buffered saline, dissociate attached cells with trypsin) on cellular uptake is not known, and further investigations are necessary to understand the reasons for the difference between in-culture measurements and conventional uptake measurements. Nevertheless, the significant correlations between the relative uptake ratios $(0.5 / 5$ and $2.5 / 5 \mathrm{mM})$ for the two types of measurements demonstrated the consistency of the relative relations between them. As many studies investigate the relative differences under certain interventions, the systematic bias between CIMR and conventional uptake may not change the results if all the investigations are performed using in-culture measurement (11). Smaller variations were achieved with in-culture measurement using CIMR than with conventional uptake experiments. Thus, CIMR provides a stable way to investigate relative changes under various interventions.

Furthermore, the uptake values obtained with CIMR are indirect estimations compared with the previous in-culture microfluidic radioassay using a loading and flushing protocol (11). Adapting the infusion profile of CIMR with flushing of the tracer allows the pure event density of cells to be measured, and the uptake values we calculated were consistent with the continuously measured CIMR uptake (Supplemental Fig. 4). This finding supports the feasibility of continuous estimation of uptake during infusion without flushing of the tracer.

Estimation of cellular pharmacokinetics requires estimation of the concentration (event density) inside and outside the cells. For the event density inside cells, the cell volume needs to be considered. However, the real cell volume is difficult to measure and was therefore estimated using the diameters of cells in the cell chamber. This step may introduce bias to the parameter estimation. Nevertheless, the estimated kinetic parameters were consistent with literature data on the same type of cancer. For a glucose concentration of $5 \mathrm{mM}$ (corresponding to the human condition), the fitted phosphorylation rates $\left(k_{3}\right)$ of this study ranged from 0.027 to $0.054 \mathrm{~min}^{-1}$ for the breast cancer cell line $\mathrm{SkBr} 3$. This range agrees with two ranges reported in the literature -0.025 to $0.061 \mathrm{~min}^{-1}$ (35) and 0.012 to $0.078 \mathrm{~min}^{-1}$ (36) - for dynamic ${ }^{18} \mathrm{~F}$-FDG PET on human breast cancer. Similarly, for a glucose concentration of $5 \mathrm{mM}$, the fitted $k_{3}$ of this study ranged from 0.031 to $0.055 \mathrm{~min}^{-1}$ for the pancreatic cancer cell line Capan-1. This range is also in line with the $k_{3}$ value of $0.041 \mathrm{~min}^{-1}$ (separating overall survival between 4 and $6 \mathrm{mo}$ ) reported in the literature (37) for ${ }^{18} \mathrm{~F}$-FDG PET on human pancreatic adenocarcinoma patients.

In the current setup, the infusion protocol (input function) is a step function, which results in a short transient response followed by a linear time-activity curve. This function may introduce bias to the estimation. Further constraints can be added by adapting the infusion protocol, such as by using a square pulse function. In Supplemental Figure 5, two different square-function infusion profiles are tested for estimation of the kinetic parameters on $\mathrm{SkBr} 3$ cells cultured using $0.5 \mathrm{mM}$ glucose. Comparing the estimations, the value of $k_{1}$ from the square function $(1.17 \pm 0.27)$ is slightly higher than that from the step function $(0.99 \pm 0.26)$. But no significant difference was observed using the unpaired $t$ test. The $k_{3}$ value from the square function $(0.13 \pm 0.08)$ is almost the same as that from the step function $(0.14 \pm 0.04)$, with no significant difference being observed. Variation was slightly larger for the square function than for the step function. There might be bias in the estimation using different infusion profiles (input functions). For the studies in this article, all $k_{4}$ values were nearly 0 . Although the square infusion profile may improve estimation and reduce bias compared with the step infusion profile, use of the square function does not bring about a significant difference for the studies without obvious dephosphorylation. However, the square function may significantly improve investigations of tracers with clear $k_{4}$ clearances.

The estimated kinetic parameters in this study cannot be directly linked to underlying physiologic behavior. We used a quantitative index - the mRNA level of corresponding proteins measured using qPCR - for comparison. However, the expression levels of the corresponding proteins may deviate from the mRNA expression levels (38). The investigated typical glucose transporter, GLUT1, and the typical phosphorylation enzyme, hexokinase-2, may not represent the overall function of multiple existing isoforms of GLUT and hexokinase. In addition to influencing protein expression, the activities of the transporters and enzymes also influence the behavior of the transport and phosphorylation (28). In contrast, the estimated kinetic parameters represent the overall effects of protein expression and activity. Furthermore, it is not always possible to fit the pharmacokinetic parameters to mRNA expression using the Michaelis-Menten equation (33) with a constant $K_{m}$ (failed for Capan-1). For each cell line, we tried to estimate $K_{m}$ for various glucose incubation conditions. We applied model fitting 
to test whether it is possible to interpret the relation between mRNA expression and kinetic parameters using a constant $K_{m}$. A change in the value of the rate constant is not expected to match the change in $V_{\max }$ especially when the concentration of the substrate (glucose level in the present case) is altered, unless the value of $K_{m}$ is much larger than the substrate concentration. Thus, it is not expected that the mRNA levels will agree with the cellular pharmacokinetics of ${ }^{18} \mathrm{~F}$-FDG in all situations.

All CIMR measurements in this study were performed under conditions of normal air, normal room temperature $\left(\sim 25^{\circ} \mathrm{C}\right)$, and normal room humidity, without the use of a dedicated incubator. Although we tried to minimize the measurement time outside the incubator, stress may still have been introduced to the cells during that time, leading to bias in the estimation of cellular uptake. Nevertheless, all environmental conditions were similar for the various runs in this study. Their variations were small compared with the variabilities due to the many other factors addressed earlier.

The CIMR system can be extended to measure positrons or electrons emitted by other tracers using the same detector as in this study $(39,40)$. The measured signal depends on the energy of the emitted positrons or electrons. For different tracers with the same positron emitter, such as the ${ }^{18} \mathrm{~F}$-labeled tracers, the proposed methods can be directly transferred. For tracers with different positron emitters, such as ${ }^{68} \mathrm{Ga}$ or ${ }^{11} \mathrm{C}$, some of the correction coefficients, such as the decay correction or the depth-dependent sensitivity correction, need to be recalculated.

\section{CONCLUSION}

A CIMR system has been developed for real-time in-culture measurement of radiotracer uptake by cells. The system estimates cellular pharmacokinetics by dynamically measuring the time course of radiotracer uptake. Initial experiments have demonstrated the reproducibility and stability of the system in capturing pharmacokinetic differences. The CIMR system provides a platform for convenient quantitative investigation of cellular physiology and pharmacokinetics.

\section{DISCLOSURE}

The costs of publication of this article were defrayed in part by the payment of page charges. Therefore, and solely to indicate this fact, this article is hereby marked "advertisement" in accordance with 18 USC section 1734 . The research leading to these results was funded by German Research Foundation (DFG) Collaborative Research Centre 824 (SFB824) and supported by the China Scholarship Council. The scientific collaboration between UCLA and TUM was supported by BaCaTec (project 17, 2012-1). No other potential conflict of interest relevant to this article was reported.

\section{ACKNOWLEDGMENTS}

We thank Birgit Blechert, Dr. Christine Bayer, and Dr. Daniela Schilling for the cell lines, Prof. Markus Essler for valuable discussions, Arianne Tran for development of the user interface software, Dr. Jan Tous (CRYTUR, spol. s r.o.) for support with the positron camera, and Dr. Armin Bieser (ibidi GmbH) for help with the microfluidic chips.

\section{REFERENCES}

1. Ong LC, Jin Y, Song IC, Yu S, Zhang K, Chow PK. 2-[ $\left[{ }^{18} \mathrm{~F}\right]-2$-deoxy-D-glucose (FDG) uptake in human tumor cells is related to the expression of GLUT-1 and hexokinase II. Acta Radiol. 2008;49:1145-1153.

2. Pratx G, Chen K, Sun C, et al. High-resolution radioluminescence microscopy of ${ }^{18}$ F-FDG uptake by reconstructing the beta-ionization track. $J$ Nucl Med. 2013;54: 1841-1846.

3. Iwamoto M, Kawada K, Nakamoto Y, et al. Regulation of ${ }^{18} \mathrm{~F}-\mathrm{FDG}$ accumulation in colorectal cancer cells with mutated KRAS. J Nucl Med. 2014;55:2038-2044.

4. Suzuki S, Kaira K, Ohshima Y, et al. Biological significance of fluorine-18-alphamethyltyrosine (FAMT) uptake on PET in patients with oesophageal cancer. $\mathrm{Br}$ J Cancer. 2014;110:1985-1991.

5. Webster JM, Morton CA, Johnson BF, et al. Functional imaging of oxidative stress with a novel PET imaging agent, ${ }^{18} \mathrm{~F}-5$-fluoro-L-aminosuberic acid. $\mathrm{J}$ Nucl Med. 2014;55:657-664.

6. Maschauer S, Prante O, Hoffmann M, Deichen JT, Kuwert T. Characterization of ${ }^{18}$ F-FDG uptake in human endothelial cells in vitro. $J$ Nucl Med. 2004;45: 455-460.

7. Saito K, Lee S, Shiuchi T, et al. An enzymatic photometric assay for 2-deoxyglucose uptake in insulin-responsive tissues and 3T3-L1 adipocytes. Anal Biochem. 2011; 412:9-17.

8. Harris T, Degani H, Frydman L. Hyperpolarized ${ }^{13} \mathrm{C}$ NMR studies of glucose metabolism in living breast cancer cell cultures. NMR Biomed. 2013;26: 1831-1843.

9. Pratx G, Chen K, Sun C, et al. Radioluminescence microscopy: measuring the heterogeneous uptake of radiotracers in single living cells. PLoS One. 2012;7: e46285.

10. Björke H, Andersson K. Automated, high-resolution cellular retention and uptake studies in vitro. Appl Radiat Isot. 2006;64:901-905.

11. Vu NT, Yu ZT, Comin-Anduix B, et al. A beta-camera integrated with a microfluidic chip for radioassays based on real-time imaging of glycolysis in small cell populations. J Nucl Med. 2011;52:815-821.

12. Wang J, Hwang K, Braas D, et al. Fast metabolic response to drug intervention through analysis on a miniaturized, highly integrated molecular imaging system. J Nucl Med. 2013;54:1820-1824.

13. Fang C, Wang Y, Vu NT, et al. Integrated microfluidic and imaging platform for a kinase activity radioassay to analyze minute patient cancer samples. Cancer Res. 2010;70:8299-8308.

14. Sha $\mathrm{W}, \mathrm{Yu} \mathrm{Z}, \mathrm{Vu} \mathrm{N}$, et al. Optimal design of a new kinetic strategy for extracting FDG transport and uptake information in microfluidic multi-chamber cell culture chip coupled with PSAPD camera. In: Yu B, ed. 2009 IEEE Nuclear Science Symposium Conference Record (NSS/MIC). Vol 1-5. Piscataway, NJ: IEEE; 2009:3936-3942.

15. Day FL, Link E, Ngan S, et al. FDG-PET metabolic response predicts outcomes in anal cancer managed with chemoradiotherapy. Br J Cancer. 2011;105: 498-504.

16. Koppenol WH, Bounds PL, Dang CV. Otto Warburg's contributions to current concepts of cancer metabolism. Nat Rev Cancer. 2011;11:325-337.

17. Pugachev A, Ruan S, Carlin S, et al. Dependence of FDG uptake on tumor microenvironment. Int J Radiat Oncol Biol Phys. 2005;62:545-553.

18. Amann T, Maegdefrau U, Hartmann A, et al. GLUT1 expression is increased in hepatocellular carcinoma and promotes tumorigenesis. Am J Pathol. 2009;174: 1544-1552.

19. Rivenzon-Segal D, Rushkin E, Polak-Charcon S, Degani H. Glucose transporters and transport kinetics in retinoic acid-differentiated T47D human breast cancer cells. Am J Physiol Endocrinol Metab. 2000;279:E508-E519.

20. Higashi T, Tamaki N, Torizuka T, et al. FDG uptake, GLUT-1 glucose transporter and cellularity in human pancreatic tumors. J Nucl Med. 1998;39:1727-1735.

21. Yen TC, See LC, Lai CH, et al. ${ }^{18}$ F-FDG uptake in squamous cell carcinoma of the cervix is correlated with glucose transporter 1 expression. J Nucl Med. 2004;45:22-29.

22. Wolf A, Agnihotri S, Micallef J, et al. Hexokinase 2 is a key mediator of aerobic glycolysis and promotes tumor growth in human glioblastoma multiforme. $J$ Exp Med. 2011;208:313-326.

23. Adams V, Kempf W, Hassam S, Briner J. Determination of hexokinase isoenzyme I and II composition by RT-PCR: increased hexokinase isoenzyme II in human renal cell carcinoma. Biochem Mol Med. 1995;54:53-58.

24. Patra KC, Wang Q, Bhaskar PT, et al. Hexokinase 2 is required for tumor initiation and maintenance and its systemic deletion is therapeutic in mouse models of cancer. Cancer Cell. 2013;24:213-228.

25. Ahn KJ, Hwang HS, Park JH, et al. Evaluation of the role of hexokinase type II in cellular proliferation and apoptosis using human hepatocellular carcinoma cell lines. J Nucl Med. 2009;50:1525-1532. 
26. Tennant DA, Duran RV, Gottlieb E. Targeting metabolic transformation for cancer therapy. Nat Rev Cancer. 2010;10:267-277.

27. Lee JD, Yang WI, Park YN, et al. Different glucose uptake and glycolytic mechanisms between hepatocellular carcinoma and intrahepatic mass-forming cholangiocarcinoma with increased ${ }^{18}$ F-FDG uptake. J Nucl Med. 2005;46: 1753-1759.

28. Rodríguez-Enriquez S, Marin-Hernandez A, Gallardo-Perez JC, MorenoSanchez R. Kinetics of transport and phosphorylation of glucose in cancer cells. J Cell Physiol. 2009;221:552-559.

29. Wong KP, Sha W, Zhang X, Huang SC. Effects of administration route, dietary condition, and blood glucose level on kinetics and uptake of ${ }^{18} \mathrm{~F}-\mathrm{FDG}$ in mice. J Nucl Med. 2011;52:800-807.

30. Wang Q, Tous J, Liu Z, Ziegler S, Shi K. Evaluation of Timepix silicon detector for the detection of ${ }^{18} \mathrm{~F}$ positrons. J Instrument. 2014;9:C05067.

31. Vaz CV, Alves MG, Marques R, et al. Androgen-responsive and nonresponsive prostate cancer cells present a distinct glycolytic metabolism profile. Int J Biochem Cell Biol. 2012;44:2077-2084.

32. Iida H, Kanno I, Miura S, Murakami M, Takahashi K, Uemura K. Error analysis of a quantitative cerebral blood flow measurement using $\mathrm{H}_{2}{ }^{15} \mathrm{O}$ autoradiography and positron emission tomography, with respect to the dispersion of the input function. J Cereb Blood Flow Metab. 1986;6:536545.
33. Sokoloff L, Reivich M, Kennedy C, et al. The $\left[{ }^{14} \mathrm{C}\right]$ deoxyglucose method for the measurement of local cerebral glucose utilization: theory, procedure, and normal values in the conscious and anesthetized albino rat. J Neurochem. 1977;28: 897-916.

34. Mitchell MJ, King MR. Fluid shear stress sensitizes cancer cells to receptormediated apoptosis via trimeric death receptors. New J Phys. 2013;15:015008.

35. Torizuka T, Zasadny KR, Recker B, Wahl RL. Untreated primary lung and breast cancers: correlation between F-18 FDG kinetic rate constants and findings of in vitro studies. Radiology. 1998;207:767-774.

36. Tseng J, Dunnwald LK, Schubert EK, et al. ${ }^{18} \mathrm{~F}-\mathrm{FDG}$ kinetics in locally advanced breast cancer: correlation with tumor blood flow and changes in response to neoadjuvant chemotherapy. J Nucl Med. 2004;45:1829-1837.

37. Epelbaum R, Frenkel A, Haddad R, et al. Tumor aggressiveness and patient outcome in cancer of the pancreas assessed by dynamic ${ }^{18} \mathrm{~F}-\mathrm{FDG}$ PET/CT. J Nucl Med. 2013;54:12-18.

38. Shim HK, Lee WW, Park SY, Kim H, Kim SE. Relationship between FDG uptake and expressions of glucose transporter type 1, type 3, and hexokinase-II in ReedSternberg cells of Hodgkin lymphoma. Oncol Res. 2009;17:331-337.

39. van Gastel R, Sikharulidze I, Schramm S, et al. Medipix 2 detector applied to low energy electron microscopy. Ultramicroscopy. 2009;110:33-35.

40. Esposito M, Jakubek J, Mettivier G, Pospisil S, Russo P, Solc J. Energy sensitive Timepix silicon detector for electron imaging. Nucl Instrum Methods Phys Res A. 2011;652:458-461. 